\title{
11
}

\section{Eight Lessons for Social Inclusion and High-Quality Sustainable Elder Care}

The inspiration for this book is derived from our commitment to the belief that sharing best practice is an essential tool to guide societies and health systems to make the adaptations needed to support the growing aging population. The case studies and interviews portray initiatives, programs, and health system innovations that can contribute to making the adaptations successful and greatly contribute to the well-being of older adults.

One of the greatest stories of the 20th century was that we doubled the life expectancy of adults. Now we need to make sure we have all the supports in place to assure not just a long life but a high quality of that long life. ${ }^{1}$ (Terry Fulmer, president of the John A. Hartford Foundation)

Many of the best practices that we describe were fueled by or made possible by value-based reimbursement policy. Value includes lower costs, better health outcomes including patient satisfaction, more access to care, and improved patient well-being. In the preceding chapters, we have seen reoccurring themes that are integral to many of the programs and initiatives that we documented. 
Here we distill our findings into eight lessons for delivery of highquality sustainable elder care and support and creating communities of social inclusion.

\section{Lesson One: The Availability of Affordable Long-Term Care Insurance Is Essential to Improve Access to Care for Older Adults}

Sadly, many people in the United States cannot afford healthcare. Older people are especially vulnerable because they use the health system the most and often cannot afford the co-payments. Additionally, Medicare does not cover dental or vision care, which leads to other health complications and impediments to well-being. The long-term care insurance industry was faced with sustainability challenges early on but has recovered in recent years, leaving higher premiums that are unaffordable by most. This is problematic because most people will need long-term care in their lifetime. The costs of such care can leave family members and the surviving spouse in poverty.

\section{Lesson Two: Person-Centered Care Is a Vital Component of High Quality Care and for Older Adults}

Long-term care providers are committing to person-centered care by embracing a more horizontal culture of teamwork steeped in the respect for the dignity of older adults. Culture change is necessary but difficult. The board, the management, and all of the staff must embrace it or it will not be sustainable. On first glance, one would think that person-centered policies require more staff and result in higher costs, but our research has shown the opposite.

We found examples of excellence in long-term care at St. John's and Beatitudes Campus with care partners who work as a team and do not 
rotate. Allen Power brought the person-centered care concept to dementia with the belief that those with dementia should not be segregated. $\mathrm{He}$ also asserts that person-centered approaches can often be used instead of antipsychotic medications. The Eden Alternative philosophy that is based in the well-being and dignity of older adults is the basis for culture change and organizational transformation that leads to person-centered policies and practices in institutional and home care.

\section{Lesson Three: Palliative Care and Social Support in the Home Is Essential to High- Quality Elder Care}

The trend toward delivering healthcare and social supports in the home and community is growing. Most people 65 and older are living with chronic illnesses, making primary and palliative care one in the same for that population. Home-based primary and palliative care can offer a better option to the expensive cycle of ED visits, hospitalizations, and skilled nursing stays that are dangerous and stressful to older adults. Additionally, insurers and providers are increasingly understanding the importance of involving the patient and the family in the care plan. Health delivery systems are reorganizing and insurers are reshaping reimbursement policy to honor the late life wishes of patients.

The Independence at Home demonstration project of Northwell Health, led by Dr. Kristofer Smith, is a glowing example of how to deliver home-based primary care to the most frail older adults that greatly improves their quality of life, honors their late life care plan, and substantially lowers the cost of care. We hope that the demonstration project will influence the Centers for Medicare and Medicaid to reimburse for Independence at Home programs and make it available to many.

Under the direction of Dr. Diane E. Meier, the Center to Advance Palliative Care has increased awareness of the large care gap that palliative care can fill-especially when delivered in all settings. The Center to Advance Palliative is also committed to encouraging all clinicians to receive geriatric care training. 
Some providers, such as Dr. Allan Teel, who are enabling their patients to age in place, connect those patients to the existing services and social supports that are available in their community. Involving the community in the daily life of older individuals makes sense. It divides the "care" burden while enabling the individual to maintain meaningful connections socially and to receive other supportive services such as meals, assistance with activities of daily living, and transportation.

\section{Lesson Four: Coordinated Primary Healthcare Is Essential to High-Quality Elder Care}

Coordinated, accessible primary care can yield better health outcomes and reduce redundant and unnecessary care. Coordinated primary care is especially effective for older individuals living with comorbidities who are receiving care in many settings and by multiple clinicians.

Dr. Erik Langhoff of the James J. Peters Veterans Health Administration demonstrated that coordinated primary care is most effective with the use of technology in care coordination across multiple specialties, electronic health record maintenance, diagnostics, telemedicine, and outcome measurement.

The patient-centered medical home model is an example of accessible and coordinated primary care that, according to Drs. Erin Giovannetti and Michael Barr with the National Committee for Quality Assurance, can produce better health outcomes, improved patient satisfaction, and reduced care costs. The National Committee for Quality Assurance is recognizing an ever-increasing number of patient-centered medical homes as providers and insurers understand the benefits.

\section{Lesson Five: Delivery of Acute and Hospital- Level Care in the Home and Community Can Lower Healthcare Costs and Improve Access and Well-Being for Older Adults}

Emergency department and hospital care is the most expensive care. Older adults use the ED and are hospitalized more than any other cohort. They are also more likely to experience adverse health events, misdiagnosis, 
functional and cognitive decline, and medical error in both settings. One way to address this issue and significantly lower the cost of care is to provide hospital-level and emergency care at home and in the community.

\section{Acute Care}

Older adults prefer to receive acute care in their homes and avoid the emotional stress of riding in an ambulance to the ED and potential subsequent hospital admission. DispatchHealth is proof that it is possible to treat acute health episodes in the home. The model, detailed by Kevin Riddleberger and Dr. Mark Prather, uses sophisticated portable diagnostic equipment and employs clinicians who are more highly trained than emergency medical technicians. DispatchHealth providers have access to the patient electronic health record system and update them in real time. The DispatchHealth team is meeting the need for acute care in senior residence communities with all levels of care. Insurers have begun to reimburse acute care in the home and community because it has shown to produce impressive cost savings, better health outcomes, greatly improved access to care, and much higher rates of patient satisfaction. DispatchHealth estimates that they have generated more than US $\$ 25$ million in cost savings since the inception of the company.

\section{Acute and Palliative Care}

Call9 is filling the acute and palliative care gap for older adults living in skilled nursing homes by providing around-the-clock technology-enabled specialized and personalized care in nursing homes. They are also enabling their patients to die with dignity in the location of their choice and with care that is aligned with the priorities of the patient and the family. Their model drastically cuts healthcare costs for their patients and improves access and patient satisfaction.

\section{Hospital Care}

Hospital-level care in the home, such as the Hospital at Home program led by Dr. Bruce Leff, produces higher levels of patient satisfaction and 
the same or better health outcomes at one third of the cost. Patients in the emergency department who meet certain criteria are transported back to their home and given care there. It is likely that the demonstration projects of this model will compel the Centers for Medicare and Medicaid Services and other insurers to make hospital care at home available through bundled payment reimbursements, enabling a larger-scale adoption in the United States.

\section{Lesson Six: Dementia Presents Challenges and Opportunities for High-Quality Care and Caregiver Support}

\section{Caregiving}

The burden of caregiving for those living with dementia is often borne by the informal caregiver. As shown by Dr. Mary Mittelman of the NYU Caregiver Intervention, the value of supporting the caregiver cannot be underestimated in regard to both cost of care and well-being. Jed Levine described the commitment of CaringKind to insuring that all individuals who are caring for people with dementia receive specific training and education because the disease presents unique challenges. Those with dementia often wish to walk around outside. As described by Elizabeth Santiago, when they become lost, there are low-tech programs to find them that can be easily and inexpensively implemented by institutions anywhere. The Wanderer's Safety Program is one such program.

\section{Institutional Settings}

Those living with dementia respond to the built environment differently. Dr. Davina Porock led efforts by institutions to use colors and visual cues that make the environments safer and more accessible. Davina described that emergency departments, hospitals, and long-term care providers are also learning that moving people with dementia from location to location can cause further disorientation and discomfort. 
Tena Alonzo and Karen Mitchell of Beatitudes Campus and Rebecca Priest with St. John's Home are creating home-like environments without the use of overhead announcement systems, alarms, and chemical or physical restraints. The environments often include animals, gardens, large common areas, and furniture (including beds) that are the same furniture one would find in non-institutional homes. To reduce clinical environmental cues further and to empower long-term care residents, most of the staff of St. John's does not wear uniforms.

In both long-term care residences, the staff is also encouraged to build meaningful relationships with the residents and their families. These relationships support the dignity of risk decisions such as whether to continue with medications that have unpleasant side effects or whether to allow a family member to walk around outside.

\section{"Behaviors"}

It would benefit caregivers and clinicians to understand that, because those living with dementia cannot communicate with words, they communicate with their actions. Dr. Allen Power, Tena Alonzo, Karen Mitchell, and Rebecca Priest support the fact that behaviors that convey unhappiness and aggravation express an unmet need. Those actions are far too often treated with antipsychotic medications and labeled as "resisting care."

Rebecca Priest and her team employ person-centered methods to identify the individual needs being expressed by their residents and design novel approaches to meet those needs. They also educate the staff and residents about the life and interests of their residents who are living with dementia. This enables everyone to see the person before his or her memory challenge. The methods of St. John's have been so successful that those who are living with dementia are able to live in community rather than in segregated areas.

Like everyone else, people with dementia have individual rhythms and habits. They do not like to live on someone else's schedule. They are not happy when they are woken up earlier than they wish, are served food when they are not hungry that they do not like, and are forced to participate in activities that they do not find enjoyable. They also do not want 
to be locked inside day and night. This displeasure can lead to aggressive or unpleasant behavior and, when expressed in the late afternoon, is labeled as sundowning. Under the guidance of Tena Alonzo and Karen Mitchell, the team of Beatitudes Campus has become so adept at understanding the individual needs, preferences, and rhythms of residents with dementia that they have ended all cases of sundowning in their memory care residences. They embrace liberalized diets and are so organizationally adaptive that they are able to allow residents to live on their own schedules.

\section{Palliative Care}

Person-centered palliative care for those living with dementia that is focused on the comfort of the patient is slowly being embraced throughout America. The model has shown to be beneficial on an institutional and organizational level while supporting the well-being of the person living with dementia. We hope that Comfort Matters ${ }^{\mathrm{TM}}$ will eventually spread to countries outside of the United States and become the standard level of dementia care we come to expect.

\section{Stigma}

We applaud the efforts of organizations such as the Dementia Action Alliance and people like Jackie and Lon Pinkowitz and Karen Love who are dedicated to confronting the stigma of those living with dementia. Caring Conversations is a simple and impactful method of convening individuals from all walks of life and in multiple professions with those living with dementia in their community. These conversations can help people see the person before his or her cognitive challenge. Rather than the deficit-based view, the whole person vision of someone who is living with dementia can have profound and lasting change on how we move forward as a society to support and embrace those with the disease.

Brian LeBlanc is a living proof that people living with dementia are powerful advocates and experts. He, Lindsay Goldman of Age-Friendly NYC, and Mia Oberlink of the AdvantAge Initiative understand that those living with dementia must be involved in all levels of policy making that deal with dementia. Doing otherwise only furthers the stigma. 


\section{Lesson Seven: It Is Imperative for Health Systems to Provide Both Social and Medical Supports with Preventative Interventions That Address the Social Determinants of Health}

Healthcare systems around the world, and most definitely the United States, need to make efforts further upstream with robust health prevention as social support measures that address the social determinants of health and treat the whole person including biopsychosocial needs. This population health concept has the potential to reduce health system costs and contribute to the well-being of all people. It is the only way forward to care for the aging population effectively and sustainably.

\section{Prevention and Care Transitions}

Evidence-based chronic disease self-management and health prevention programs, such as those described by the Partners in Care Foundation founder June Simmons, have proven to be impactful on health outcomes and healthcare costs. Additionally, employing varying levels of clinical care and social supports can enable a safe transition from the hospital directly to the home that greatly improves patient well-being and lowers healthcare costs.

\section{Function}

Under the leadership of Sarah Szanton, the CAPABLE program is proof positive that it is possible to combine social and medical supports to improve the functional capacities of the most frail, community-dwelling older adults. Sarah taught us that goal-oriented, restorative care is most effective when the goals of the program participant are honored as the top priority because people are more willing to challenge themselves to meet a goal that is meaningful to them. 


\section{Lesson Eight: Social Inclusion and the Opportunity to Live a Purposeful Life Are Essential to the Happiness and Well-Being of Older Adults}

Social inclusion represents upstream physical and mental health prevention. Inclusion can delay frailty and mitigate loneliness, isolation, and depression. Social inclusion also helps dispel the stigma of aging. Public and private organizations and communities are building inclusive and accessible environments for older people. Some community members have joined together to build socially connected hubs where people of all ages can remain engaged and supported.

Dr. Paul Tang and his team at linkAges created one such hub in the model of a timebank where community members help each other out on a trade basis. Physicians in the Sutter Health practice can write a prescription for timebanking when they see the need for social inclusion. This is a fine example of providing both health and social support under the same roof.

Emi Kiyota leads Ibasho as they support the development of community-led social hubs called cafés. The three cafés around the world serve as a form of community resilience after natural disasters. They are community-run, self-sustaining meeting places for people of all ages and abilities.

\section{Housing}

Housing can enrich the lives of older adults through multigenerational connections, inclusive built environments, and lifelong learning. Communities such as St. John's and Beatitudes Campus that provide space and support for older adults to create their own clubs and civic activities built on their life experience and passions send a strong message that older individuals still have much to give and can have lasting impact on the younger generation, their community, and all levels of policy. 
As shown by the Naturally Occurring Retirement Communities and the Village to Village movement there are many successful examples of neighborhoods whose members joined together to receive social supports and services and healthcare collectively in the community. These communities will likely continue to grow with the aging population.

Joani Blank is an older person who embraces community living in the form of cohousing. Cohousing structures are designed to enhance community inclusion and productive cooperation with shared spaces and shared meals and projects. The multigenerational nature of cohousing enables fellow residents to form meaningful relationships with their neighbors of all ages.

Led by Anne Doyle, Lasell Village was designed with the dedication to lifelong learning and intergenerational connections. The retirement community is on the campus of Lasell College and supports continual opportunities for lifelong learning at the college and in the community. Lasell Village is also home to a nursery school. Some residents volunteer at the school and others enjoy the children involved in their daily activities throughout Village.

\section{Age-Inclusive Cities}

Led by Lindsay Goldman, Age-Friendly NYC is proof that environments that are accessible to people of all ages and abilities are stronger, more resilient, and more economically secure. Older community members must be involved in all stages of planning and implementing age-inclusive initiatives. This was especially impactful with the early efforts of AgeFriendly NYC when they conducted town halls around the city to learn directly from older individuals what was needed to make the city accessible and to enable them to live engaged productive lives. Lindsay Goldman asserts that, to have the greatest impact and remain sustainable, age-inclusive planning should also include public and private entities from a variety of sectors while remaining true to the mantra of "Age in Everything."

An important aspect of inclusive cities is the availability for older people to remain active in the workforce in whatever capacity they desire or 
need. Employment keeps people engaged as productive members of society. It also keeps them socially and physically active. In many cases employment enables older adults to earn much needed income. Older adults also help fill the growing workforce shortage. Under the leadership of Ruth Finkelstein, the Age Smart Employer initiative seeks to honor employers who are able to attract and retain older employees through training, job modification, creating mentor-type relationships, and offering part-time employment and step-down retirement options.

Including individuals of all ages in civic and community life helps to dispel the harmful stigma of aging. Through the program, Exceeding Expectations, Ruth Finkelstein and journalist Dorian Block use storytelling to dispel the aging stigma. They profile a cross section of adults in New York City who have lived longer than the life expectancy of the city and are living vibrant active lives.

\section{Looking Forward}

The United States might not be the first country one would expect to find exceptional care and support for older adults. We were pleasantly surprised to find such examples ourselves. The only way around the existing, inefficient, and expensive health system is with an innovative pioneering spirit that many Americans still hold dear. It is with disappointment that we must acknowledge that the models of excellence are the exception and not the rule. We are encouraged by our research that this is changing, albeit slowly.

We hope that this book serves as inspiration and incentive to health providers and those dedicated to optimal aging and well-being of older adults around the world. We believe, however, that the strongest and most lasting transformations will be driven by the stakeholders-the older adults who, as baby boomers, changed almost every system they touched from their active civic engagement to their demand for convenience and person-centered service. We have seen that excellence is possible and must all unite to make it standard practice. 


\section{Additional Reading}

We would like to mention that our office in Sweden conducted a series of interviews with similar focus areas including long-term care, care coordination, mobile health, home health, person-centered care, caregiver support, technology, dementia care, and housing models for those living with dementia. The interviews have been distilled into takeaways and analyses that are available in a new book entitled Aging with Dignity. The book will be available on Amazon soon. For more information on Aging with Dignity: http://accessh.org/agingwithdignity2/

\section{Note}

1. Hafner, K. (2016). As population ages, where are the geriatricians? New York Times. http://health.usnews.com/health-news/patient-advice/articles/2015/04/21/doctor-shortage-who-will-take-care-of-the-elderly. Accessed April 2017.

Open Access This chapter is licensed under the terms of the Creative Commons Attribution 4.0 International License (http://creativecommons.org/licenses/ by/4.0/), which permits use, sharing, adaptation, distribution and reproduction in any medium or format, as long as you give appropriate credit to the original author(s) and the source, provide a link to the Creative Commons licence and indicate if changes were made.

The images or other third party material in this chapter are included in the chapter's Creative Commons licence, unless indicated otherwise in a credit line to the material. If material is not included in the chapter's Creative Commons licence and your intended use is not permitted by statutory regulation or exceeds the permitted use, you will need to obtain permission directly from the copyright holder.

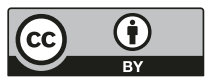

